\title{
An Algorithm for Generating Classification Rules Based on Extended Function Dependency
}

\author{
Xiaoping Zhang, Fengzhan Tian, and Houkuan Huang \\ School of Computer \& Information Technology, \\ Beijing Jiaotong University, Beijing 100044, P.R. China \\ xiao_ping_zhang@126.com, \{fzhtian,hkhuang\}@bjtu.edu.cn
}

\begin{abstract}
Classification is an important task in the fields of data mining and pattern recognition. Now there have been many algorithms for this task, while most of them do not focus on the application in databases. In this paper we extend the definition of function dependency, prove the properties of the extended function dependency, and on this basis propose an algorithm for classification. According to the two theorems in the paper, our algorithm is complete that means it can find all the classification rules from the database. At last, we demonstrate our algorithm by an example that shows the validity of our algorithm.
\end{abstract}

Keywords: Data mining, Classification, Function Dependency, Scope.

\section{Introduction}

Classification is an important task in the fields of data mining and pattern recognition $^{[1,2]}$ and there have been developed many algorithms used for this task ${ }^{[3]}$ up to now. At present, the most used classification methods include decision tree method (such as $\mathrm{C} .5^{[4]}$, SLI algorithm ${ }^{[5]}$ and SPRIN algorithm ${ }^{[6]}$ ), Bayesian classifiers (such as Naive Bayes ${ }^{[7]}$, TAN $^{[8,9]}$ etc.), artificial neural network ${ }^{[10]}$, classification algorithm based on association rules ${ }^{[11]}$, rough $\operatorname{set}^{[12]}$ and so on. Although these algorithms are used successfully in many problems, they all use the strategy of handling the dataset in main memory that is hard to integrate with database system. When the dataset is too large to be stored in memory, the above methods can't be applied. So it is very important to study classification techniques oriented to database system.

At present, classification algorithms oriented to database system mainly include MIND algorithm ${ }^{[13]}$ and GAC-RDB algorithm ${ }^{[14]}$. MIND builds classifiers using decision tree method, compute the class distribution information of the datasets of non-leaf nodes using UDF method and partition the dataset. MIND algorithm is apt to integrate with database system, but the UDF is achieved by advanced program language that can't utilize the query optimization mechanism of the database system. So it is very complex to program and maintain UDF. In addition, the function achieved by SQL statements in MIND algorithm is very simple, but the way of using SQL statements is very complex. GAC-RDB is a kind of classification algorithm using SQL statements. Its advantage is to use the standard group aggregation 
statements which can take advantage of the query processing function of database system so as to improve its efficiency and expandability. Also, the algorithm does not need to transform the dataset to the format of transaction database and to scan the dataset repeatedly. Furthermore the algorithm is apt to be programmed and integrated with database system. But the algorithm still needs to be improved in some aspects, such as how to determine automatically the parameters in the algorithm and how to improve the feature selection method etc.

In this paper, we use the idea of discernible matrix in rough set ${ }^{[15]}$ and Granule calculating $^{[16]}$ for reference and extend the definition of function dependency. And then, we prove the properties of scope of the extended function dependency and on this basis propose a classification algorithm. Our algorithm has the following advantages. It can obtain the Boolean matrixes representing the function dependency relations by scanning database only once. Using logical operation 'Or' on Boolean matrixes, we can get all the classification rules. The algorithm is efficient because of the use of Boolean operations. In addition, the algorithm can generate all the classification rules without requiring manual input parameters. The two theorems in the paper guarantee the completeness of our algorithm. Finally, the generated classification rules are represented as the form of function dependency and easy to understand.

\section{Function Dependency and Its Extension}

\subsection{The Definition of Function Dependency}

Definition 1. Let $R$ is a relational schema, $X, Y \subseteq R, X \rightarrow Y$ represents a function dependency, $r$ is a relation of $R$. $r$ fits function dependency $X \rightarrow Y$ if and only if $u_{1}[X]=u_{2}[X]$ implies $u_{1}[Y]=u_{2}[Y]$ for $\forall u_{1}, u_{2} \in r$. An equivalent description is as follow: $r$ fits function dependency $X \rightarrow Y$ if and only if for $\forall\left(u_{1}, u_{2}\right) \in r \times r$, $\left(u_{1}[X]=u_{2}[X]\right) \Rightarrow\left(u_{1}[Y]=u_{2}[Y]\right)$ is true.

There are two conditions of $\left(u_{1}[X]=u_{2}[X]\right) \Rightarrow\left(u_{1}[Y]=u_{2}[Y]\right)$ being true. One is $u_{1}[X] \neq u_{2}[X]$; the other is $u_{1}[X]=u_{2}[X]$ and $u_{1}[Y]=u_{2}[Y]$. Definition 1 shows that in classical relation theory, relation $r$ fitting function dependency $X \rightarrow Y$ indicates that each pair $\left(u_{1}, u_{2}\right)$ in $r \times r$ satisfies implication $\left(u_{1}[X]=u_{2}[X]\right) \Rightarrow\left(u_{1}[Y]=u_{2}[Y]\right)$. Next we extend the above definition and allow part of pairs $\left(u_{1}, u_{2}\right)$ in $r \times r$ to satisfy $\left(u_{1}[X]=u_{2}[X]\right) \Rightarrow\left(u_{1}[Y]=u_{2}[Y]\right)$. We name the set of this part of pairs $\left(u_{1}, u_{2}\right)$ as the scope of function dependency $X \rightarrow Y$.

\subsection{The Definition of Extended Function Dependency}

Definition 2. Let $R$ is a relational schema, $r=\left\{u_{1}, \ldots u_{n}\right\}$ is a relation of $R$, $X, Y \subseteq R$, the scope of function dependency $X \rightarrow Y$ on relation $r$ is denoted with $F_{X, Y}$, which is a subset of $r \times r$ : 


$$
F_{X, Y}=\left\{\left(u_{i}, u_{j}\right) \mid u_{i}, u_{j} \in r \wedge\left(\left(u_{i}[X]=u_{j}[X]\right) \Rightarrow\left(u_{i}[Y]=u_{j}[Y]\right)\right)\right\}
$$

According to classical relation theory, if $r$ fits $X \rightarrow Y$, then $F_{X, Y}=r \times r$, and if $r$ does not fits $X \rightarrow Y$, then $F_{X, Y} \subset r \times r$.

Definition 3. Let $R$ is a relational schema, $r=\left\{u_{1}, \ldots u_{n}\right\}$ is a relation of $R, X, Y \subseteq R$, the Boolean matrix $T$ of function dependency $X \rightarrow Y$ on relation $r$ is:

$$
t_{i j}=\left\{\begin{array}{ll}
1 & \left(u_{i}, u_{j}\right) \in F_{X, Y} \\
0 & \text { else }
\end{array} \quad(i, j=1, \ldots, n)\right.
$$

In Boolean matrix $T$, if all the values of a whole line are 1, we call this line as 'all 1 line'. And then we define the set of elements corresponding to the 'all 1 line' as scope of function dependency $X \rightarrow Y$.

For the analysis purpose, if we regard the table 1 as original database to generate classification rules and $D$ as class attribute which are taken from the records in breastcancer-wisconsin_data of $\mathrm{UCI}^{[17]}$ and then the reduplicate record is removed, then we can regard $\left\{u_{1}, u_{2}, u_{3}\right\}$ as class one, $\left\{u_{4}, u_{5}, u_{6}\right\}$ as class two. We can extend function dependency to applications of classification.

Table 1. An example of a relation $r$

\begin{tabular}{ccccc}
\hline$r$ & $A$ (Clump thickness) & $\begin{array}{c}B \text { (Uniformity of } \\
\text { Cell Size })\end{array}$ & $C$ (Mitoses) & $D$ (Class) \\
\hline$u_{1}$ & 2 & 1 & 1 & 2 \\
$u_{2}$ & 3 & 1 & 1 & 2 \\
$u_{3}$ & 2 & 1 & 1 & 2 \\
$u_{4}$ & 10 & 1 & 1 & 4 \\
$u_{5}$ & 2 & 10 & 4 & 4 \\
$u_{6}$ & 2 & 7 & 1 & 4 \\
\hline
\end{tabular}

\subsection{The Properties of the Scope of the Extended Function Dependency}

Theorem 1. Let $R$ is a relational schema, $r$ is a relation of $R, X_{1}, X_{2}, Y \subseteq R$, then $F_{X_{1} \cup X_{2}, Y}=F_{X_{1}, Y} \cup F_{X_{2}, Y}$.

Proof. We only need to prove the following two propositions :

(1) For $\forall\left(u_{1}, u_{2}\right) \in r \times r$, if $\left(u_{i}, u_{j}\right) \in F_{X_{1} \cup X_{2}, Y}$, then $\left(u_{i}, u_{j}\right) \in F_{X_{1}, Y}$ or $\left(u_{i}, u_{j}\right) \in F_{X_{2}, Y}$, whose equivalent proposition is that if $\left(u_{i}, u_{j}\right) \notin F_{X_{1}, Y}$ and $\left(u_{i}, u_{j}\right) \notin F_{X_{2}, Y}$, then $\left(u_{i}, u_{j}\right) \notin F_{X_{1} \cup X_{2}, Y}$.

(2) For $\forall\left(u_{1}, u_{2}\right) \in r \times r$, if $\left(u_{i}, u_{j}\right) \in F_{X_{1}, Y}$ or $\left(u_{i}, u_{j}\right) \in F_{X_{2}, Y}$, then $\left(u_{i}, u_{j}\right) \in F_{X_{1} \cup X_{2}, Y}$. 
The proof of the first proposition is as follows. According to $\left(u_{i}, u_{j}\right) \notin F_{X_{1}, Y}$ and the definition 2, we get $u_{i}\left[X_{1}\right]=u_{j}\left[X_{1}\right]$ and $u_{i}[Y] \neq u_{j}[Y]$. According to $\left(u_{i}, u_{j}\right) \notin F_{X_{2}, Y}$, we know $u_{i}\left[X_{2}\right]=u_{j}\left[X_{2}\right]$ and $u_{i}[Y] \neq u_{j}[Y]$. To sum up, we can infer that $u_{i}\left[X_{1} \cup X_{2}\right]=u_{j}\left[X_{1} \cup X_{2}\right]$ and $u_{i}[Y] \neq u_{j}[Y]$, that means $\left(u_{i}, u_{j}\right) \notin F_{X_{1} \cup X_{2}, Y}$. So the first proposition is proved.

The proof of the second proposition is given as following. When there is $\left(u_{i}, u_{j}\right) \in F_{X_{1}, Y}$, according to the definition 2, there are two following cases. One case is $u_{i}\left[X_{1}\right] \neq u_{j}\left[X_{1}\right]$. Then there is $u_{i}\left[X_{1} \cup X_{2}\right] \neq u_{j}\left[X_{1} \cup X_{2}\right]$ and then we know $\left(u_{i}, u_{j}\right) \in F_{X_{1} \cup X_{2}, Y}$ according to the definition 2. The other is $u_{i}\left[X_{1}\right]=u_{j}\left[X_{1}\right]$ and $u_{i}[Y]=u_{j}[Y]$, Whether $u_{i}\left[X_{1} \cup X_{2}\right]=u_{j}\left[X_{1} \cup X_{2}\right]$ or not, we can have $\left(u_{i}, u_{j}\right) \in F_{X_{1} \cup X_{2}, Y}$.

When there is $\left(u_{i}, u_{j}\right) \in F_{X_{2}, Y}$, in the same way, we can prove $\left(u_{i}, u_{j}\right) \in F_{X_{1} \cup X_{2}, Y}$. So we conclude that if $\left(u_{i}, u_{j}\right) \in F_{X_{1}, Y}$ or $\left(u_{i}, u_{j}\right) \in F_{X_{2}, Y}$, there is $\left(u_{i}, u_{j}\right) \in F_{X_{1} \cup X_{2}, Y}$. The second proposition is proved. Therefore, the original proposition is proved.

Theorem 2. Let $R=\left\{A_{1}, \cdots, A_{n}, D\right\}$ is a relational schema, where $A_{1}, \cdots, A_{n}$ are conditional attributes and $D$ is a class attribute, $r$ is relation of $R, u \in r, X \subseteq R$. If for $\forall u_{i} \in r$, there is $\left(u, u_{i}\right) \in F_{X, D}$ and for each $X_{1} \subset X$, there exist $u_{i} \in r$ which does not satisfy $\left(u, u_{i}\right) \in F_{X_{1}, D}$, then $(X=u[X]) \Rightarrow(D=u[D])$ is a classification rule.

Proof. For each $u_{i} \in r$, according to $\left(u, u_{i}\right) \in F_{X, D}$, we know $u[X] \neq u_{i}[X]$ or $u[X]=u_{i}[X]$ and $u[D]=u_{i}[D]$. That means for each element $v$ whose value is $u[X]$ on $X$, there is $v[D]=u[D]$, namely $(X=u[X]) \Rightarrow(D=u[D])$. And because for each $X_{1} \subset X$, there is $\left(X_{1}=u\left[X_{1}\right]\right) \nRightarrow(D=u[D])$, so we know $(X=u[X]) \Rightarrow(D=u[D])$ is a classification rule.

\section{An Algorithm for Generating Classification Rules Based on Scope of Extended Function Dependency}

On the basis of Theorem 1 and Theorem 2, we propose an algorithm for generating classification rules based on scope of extended function dependency, whose procedure is as follows.

Input: Relational schema $R=\left\{A_{1}, \cdots, A_{n}, D\right\}$ and relation $r$ of $R$, where $A_{1}, \cdots, A_{n}$ are conditional attributes and $D$ is a class attribute

Output: A set of all classification rules $S$

Suppose the reduplicate records are removed before following step. 
Step 1. For conditional attributes $A_{1}, \cdots, A_{n}$, calculate the Boolean matrixes of $F_{A_{1}, D}, \ldots, F_{A_{n, D}}$ in turn. For each matrix, each 'all 1 line' $u$ can generate a classification rule, $\left(A_{i}=u\left[A_{i}\right]\right) \Rightarrow(D=u[D])$. Add it to set $S$.

Step 2. Let $i=2, T=\left\{F_{A_{1}, D}\right\}$.

Step 3. Let $T^{\prime}=T \oplus F_{A_{i}, D}$, where

$$
T \oplus F_{A_{i}, D}=\left\{F_{X \cup\left\{A_{i}\right\}, D} \mid F_{X \cup\left\{A_{i}\right\}, D}=F_{X, D} \cup F_{A_{i}, D} \text { and } F_{X, D} \in T\right\}
$$

Step 4. Check the 'all 1 lines' of each $F_{X^{\prime}, D}$ in $T^{\prime}$ (here $X^{\prime}=X \cup A_{i}$ ). If the line is included neither in $T$ nor in $F_{A_{i}, D}$, then we call the line as a new 'all 1 line'. It can generate a classification rule, $\left(X^{\prime}=u\left[X^{\prime}\right]\right) \Rightarrow(D=u[D])$. Add the rule to set $S$.

Step 5. Let $T=T \cup T^{\prime} \cup\left\{F_{A_{i}, D}\right\}$.

Step 6. Let $i=i+1$. If $i \leq n$, then go step 3; otherwise exit.

Suppose that the numbers of records and attributes in the database are $m$ and $n$ respectively. The Step 1 in the above procedure need to scan the database only once and then get $F_{A_{1}, D}, \ldots, F_{A_{n, D}}$, whose time complexity is $\mathrm{O}\left(m^{2} *_{n}\right)$. The time complexity is also $\mathrm{O}\left(m^{2} * n\right)$ to find the 'all 1 lines' in $F_{A_{i}, D}$. In step 3, the time complexity of circling calculation of $T^{\prime}=T \oplus F_{A_{i}, D}$ is $\mathrm{O}\left(2^{n} * m^{2}\right)$. In step 4 , the time complexity is $\mathrm{O}\left(2^{n} * m^{2}\right)$ at worst case to check new 'all 1 lines' in each $F_{X^{\prime}, D}$ and then generate classification rules. So the time complexity of the whole procedure is $\mathrm{O}\left(m^{2} * n\right)+\mathrm{O}\left(2^{n} * m^{2}\right)$.

\section{A Computation Example}

Suppose $R=\{A, B, C, D\}$ and relation $r$ of $R$ is shown in Table 1. Assume $D$ is a class attribute. According to the above algorithm, the steps to generate classification rules are as follows.

Step 1. Firstly generate the Boolean matrixes of function dependency $A \rightarrow D$, $B \rightarrow D$ and $C \rightarrow D$, which are denoted as table 2, table 3 and table 4 respectively. According to 'all 1 lines' in table 2, we know that $F_{A, D}$ contains $u_{2}, u_{4}$. So we have classification rules $\left(A=u_{2}[A]\right) \Rightarrow\left(D=u_{2}[D]\right)$ and $\left(A=u_{4}[A]\right) \Rightarrow\left(D=u_{4}[D]\right)$, which means $(A=3) \Rightarrow(D=2)$ and $(A=10) \Rightarrow(D=4)$. In the same way, we can get classification rule $(B=10) \Rightarrow(D=4)$ and $(B=7) \Rightarrow(D=4)$. And also according to the three 'all 1 lines' $u_{5}$ in table $4 \cdot$ we can get classification rule $(C=4) \Rightarrow(D=4)$. So, $S=\{(A=3) \Rightarrow(D=2),(A=10) \Rightarrow(D=4),(B=10)$ $\Rightarrow(D=4),(B=7) \Rightarrow(D=4),(C=4) \Rightarrow(D=4)\}$.

Step 2. Let $i=2, T=\left\{F_{A, D}\right\}$.

Step 3. Let $T^{\prime}=T \oplus F_{B, D}=F_{A B, D}$, which is denoted as table 5, where all the new 'all 1 lines' are denoted by bold font. 
Step 4. The new 'all 1 lines' in table 5 are $u_{1}, u_{3}$. So we have the following rules $(A B=\langle 2,1\rangle) \Rightarrow(D=2)$. Therefore, $S=S \bigcup\{(A B=\langle 2,1\rangle) \Rightarrow(D=2)\}$.

Step 5. Let $T=\left\{F_{A, D}, F_{A B, D}, F_{B, D}\right\}$.

Step 6. $i=i+1=3$, go back to Step 3 of the algorithm and recalculate $T$ and $S$, whose scope of function dependency are $F_{A C, D}, F_{B C, D}$ and $F_{A B C, D}$ respectively. The computation process is as same as the above, so we do not give the details here for the sake of space. At last, we get the following set of all the classification rules:

$$
\begin{aligned}
& S=\{\quad(A=3) \Rightarrow(D=2) \quad, \quad(A=10) \Rightarrow(D=4) \quad, \quad(B=10) \Rightarrow(D=4) \quad, \quad(B=7) \\
& \Rightarrow(D=4),(C=4) \Rightarrow(D=4),(A B=\langle 2,1\rangle) \Rightarrow(D=2)\} .
\end{aligned}
$$

Table 2. Boolean matrix of function dependency $A \rightarrow D$

\begin{tabular}{ccccccc}
\hline$F_{A, D}$ & $u_{1}$ & $u_{2}$ & $u_{3}$ & $u_{4}$ & $u_{5}$ & $u_{6}$ \\
\hline$u_{1}$ & 1 & 1 & 1 & 1 & 0 & 0 \\
$\boldsymbol{u}_{\mathbf{2}}$ & $\mathbf{1}$ & $\mathbf{1}$ & $\mathbf{1}$ & $\mathbf{1}$ & $\mathbf{1}$ & $\mathbf{1}$ \\
$u_{3}$ & 1 & 1 & 1 & 1 & 0 & 0 \\
$\boldsymbol{u}_{\mathbf{4}}$ & $\mathbf{1}$ & $\mathbf{1}$ & $\mathbf{1}$ & $\mathbf{1}$ & $\mathbf{1}$ & $\mathbf{1}$ \\
$u_{5}$ & 0 & 1 & 0 & 1 & 1 & 1 \\
$u_{6}$ & 0 & 1 & 0 & 1 & 1 & 1 \\
\hline
\end{tabular}

Table 3. Boolean matrix of function dependency $B \rightarrow D$

\begin{tabular}{ccccccc}
\hline$F_{B, D}$ & $u_{1}$ & $u_{2}$ & $u_{3}$ & $u_{4}$ & $u_{5}$ & $u_{6}$ \\
\hline$u_{1}$ & 1 & 1 & 1 & 0 & 1 & 1 \\
$u_{2}$ & 1 & 1 & 1 & 0 & 1 & 1 \\
$u_{3}$ & 1 & 1 & 1 & 0 & 1 & 1 \\
$u_{4}$ & 0 & 0 & 0 & 1 & 1 & 1 \\
$\boldsymbol{u}_{\mathbf{5}}$ & $\mathbf{1}$ & $\mathbf{1}$ & $\mathbf{1}$ & $\mathbf{1}$ & $\mathbf{1}$ & $\mathbf{1}$ \\
$\boldsymbol{u}_{\mathbf{6}}$ & $\mathbf{1}$ & $\mathbf{1}$ & $\mathbf{1}$ & $\mathbf{1}$ & $\mathbf{1}$ & $\mathbf{1}$ \\
\hline
\end{tabular}

Table 4. Boolean matrix of function dependency $C \rightarrow D$

\begin{tabular}{ccccccc}
\hline$F_{B, D}$ & $u_{1}$ & $u_{2}$ & $u_{3}$ & $u_{4}$ & $u_{5}$ & $u_{6}$ \\
\hline$u_{1}$ & 1 & 1 & 1 & 0 & 1 & 0 \\
$u_{2}$ & 1 & 1 & 1 & 0 & 1 & 0 \\
$u_{3}$ & 1 & 1 & 1 & 0 & 1 & 0 \\
$u_{4}$ & 0 & 0 & 0 & 1 & 1 & 1 \\
$\boldsymbol{u}_{5}$ & $\mathbf{1}$ & $\mathbf{1}$ & $\mathbf{1}$ & $\mathbf{1}$ & $\mathbf{1}$ & $\mathbf{1}$ \\
$u_{6}$ & 0 & 0 & 0 & 1 & 1 & 1 \\
\hline
\end{tabular}


Table 5. Boolean matrix of function dependency $A B \rightarrow D$

\begin{tabular}{ccccccc}
\hline$F_{A B, D}$ & $u_{1}$ & $u_{2}$ & $u_{3}$ & $u_{4}$ & $u_{5}$ & $u_{6}$ \\
\hline $\boldsymbol{u}_{\mathbf{1}}$ & $\mathbf{1}$ & $\mathbf{1}$ & $\mathbf{1}$ & $\mathbf{1}$ & $\mathbf{1}$ & $\mathbf{1}$ \\
$u_{2}$ & 1 & 1 & 1 & 1 & 1 & 1 \\
$\boldsymbol{u}_{\mathbf{3}}$ & $\mathbf{1}$ & $\mathbf{1}$ & $\mathbf{1}$ & $\mathbf{1}$ & $\mathbf{1}$ & $\mathbf{1}$ \\
$u_{4}$ & 1 & 1 & 1 & 1 & 1 & 1 \\
$u_{5}$ & 1 & 1 & 1 & 1 & 1 & 1 \\
$u_{6}$ & 1 & 1 & 1 & 1 & 1 & 1 \\
\hline
\end{tabular}

\section{Conclusion}

In this paper, we extend the definition of function dependency, describe the concept of scope of function dependency and prove its properties. Based on the above properties, we put forward an algorithm for generating all the classification rules. In our algorithm, the dataset, Boolean matrixes and classification rules are all stored in database, so our algorithm can be applied to classification tasks in case of large-scale datasets. The algorithm is efficient because most of calculate is Boolean operation on Boolean matrixes. Our algorithm generates all the classification rules, but some classification rules are complex and are not typical. Next, we will do research on how to evaluate, merge and tailor the rule set to generate simple and effective rules. Our algorithm provides a new idea for research of classification method.

\section{Acknowledgments}

This work is supported by NSF of China under grant NO. 60503017, Beijing Nova Programme under grant NO. 2006A17 as well as Science Foundation of Beijing Jiaotong University under Grant No. 2005SM012.

Thank anonymous readers for helpful comments.

\section{References}

1. Han J. W., Fu Y. J., Wang W. et al.: DBMiner: A System for Mining Knowledge in Large Relational Database. In: Proceedings of the $2^{\text {nd }}$ International Conference on Knowledge Discovery and Data Mining. CA, USA(1996) 250-255

2. Agrawal R., Mehta M., Shafer J. et al.: The Quest Data Mining System. In: Proceedings of the 2nd International Conference on Data Mining and Knowledge Discovery, Portland, Oregon (1996) 244-249

3. Liu H. Y.: Research and Implementation of a Fast and Scalable Classification System. Beijing: Tsinghua University(2000)

4. Quinlan J. R.: C4.5: Programs for Machine Learning. San Mateo. California: Morgan Kaufmann (1993) 
5. Mehta M., Agrawal R., Rissanen J.: SLIQ: A fast scalable classifier for data mining. Lecture Notes in computer Science, Proc of the 5th International Conference on Extending Database Tech. Avignon. France(1996) 18-33

6. Shafer J. C., Agrawal R, Mehta M.: SPRINT: A scalable parallel classifier for data mining. Proc of the 22nd Int Conf on Very Large Databases. Mumbai Bombay.India(1996)

7. Elkan C.: Boosting and naïve bayesian learning. In Technical Report CS97-557, Dept. of Computer Science and Engineering, Univ. Calif. At San Diego(1997)

8. Friedman N. ,Geiger D. and Goldszmidt M.: Bayesian network classifier. Machine Learning. Vol. 29. (1997) 131-163

9. Meretakis D. and Wuthrich B.: Extending Naive Bayes classifiers using long itemsets. Chaudhuri S.Proceedings of $5^{\text {th }}$ International Conference on Knowledge Discovery and Data Mining. USA: AAAI Press(1999)295-301

10. Weiss S. M. and Kulikowski C. A.: Computer Systems That Learn: Classification and Prediction Methods from Statistics. Neural Nets, Machine Learning, and Expert Systems. San Mateo, CA: Morgan Kaufmann(1991)

11. Liu B. Hsu W. Ma Y. : Integrating classification and association rule mining. Agrawal R Proc of the 4th Int Conf on Knowledge Discovery and Data Mining. NY.USA: AAAI Press(1998)80-86

12. $\mathrm{Hu} \mathrm{X}$. H.,Cercone N.: Learing in relational database: a Rough Set approach. Computational Intelligence Vol 11. (1995) 323-338

13. WANG M., Iyer B., Vitter J. S.: Scalable mining for classification rules in relational databases. Eaglestone B. Desai B C, SHAO Jianhua.Proc of the 1998 Int Database Eng and Appl Syrup Cardiff,Wales.UK: IEEE Computer Society(1998) 58-67

14. Lu H. J. Liu H. Y.: Decision tables: scalable calssification exploring RDBMS capabilities. Proc $26^{\text {th }}$ Int Conf on Very Large Databases. Cairo.Egypt (2000)373-384

15. Skowron A. ,Rauszer C.: The discernibility matrices and functions in information system. In : Slowinsk; Red. Intelligent Decision Support-Handbook of Applictions and Advances of the Rough Set Theory, Kluwer Academic Publishers(1992) 331-362

16. Liu Q. and Jiang S. L.: Reasoning about Information Granulaes Based on the Rough Logic. LNAI 2375, Springer(2002) 139-143

17. Bennett K. P., Mangasarian O. L.: Robust linear programming discrimination of two linearly inseparable sets, Optimization Methods and Software 1, Gordon \& Breach Science Publishers(1992) 23-34 